TRANSACTIONS OF THE

AMERICAN MATHEMATICAL SOCIETY

Volume 358, Number 7, Pages 3207-3217

S 0002-9947(06)03838-4

Article electronically published on February 20, 2006

\title{
MODULI OF CURVES AND SPIN STRUCTURES VIA ALGEBRAIC GEOMETRY
}

\author{
GILBERTO BINI AND CLAUDIO FONTANARI
}

\begin{abstract}
Here we investigate some birational properties of two collections of moduli spaces, namely moduli spaces of (pointed) stable curves and of (pointed) spin curves. In particular, we focus on vanishings of Hodge numbers of type $(p, 0)$ and on computations of Kodaira dimension. Our methods are purely algebro-geometric and rely on an induction argument on the number of marked points and the genus of the curves.
\end{abstract}

\section{INTRODUCTION}

In the last decade, ideas from physics have reinvigorated the interest in moduli spaces. As a consequence, the problem of investigating their geometry has become more and more intriguing. Usually, such spaces parametrize (pointed) genus $g$ complex curves with, possibly, extra structures defined on $C$, such as a morphism to a fixed variety or a spin structure. Moreover, they may usually be viewed as objects in different categories, specifically as stacks, orbifolds, or $\mathbb{Q}$-factorial projective schemes.

In the present paper, we focus on two classical moduli spaces, namely $\overline{\mathcal{M}}_{g, n}$ and $\bar{S}_{g, n}$. We recall that the former one parametrizes $n$-pointed genus $g$ stable curves and the latter one parametrizes $n$-pointed quasi-stable curves $C$ of genus $g$ with a line bundle whose second tensor power is isomorphic to the dualizing sheaf $\omega_{C}$; see Section 3 for precise definitions. Throughout, we regard them as projective normal varieties over the field of complex numbers.

It is impossible to condense in a short paragraph all the various results and open problems in connection with these two varieties. We refer the reader to, for instance, [10, [11, 21] and the references cited therein. We just mention here that some birational invariants of $\overline{\mathcal{M}}_{g, n}$ are only conjecturally known, such as the Kodaira dimension of $\overline{\mathcal{M}}_{g}, 17 \leq g \leq 23$, or partially computed, such as $(p, 0)$-type Hodge numbers (cf. [13, 14], 15], and [27]). Furthermore, little is known about $\bar{S}_{g, n}$. As a finite ramified covering of $\overline{\mathcal{M}}_{g, n}$, its geometry is even more complicated. Recent results on the topology and the rational cohomology can be found, for example, in [7, 8], 9], 19, 20. For a different, stack-theoretic approach see 11, 23, 24.

As shown by Enrico Arbarello and Maurizio Cornalba in [3, a purely algebrogeometric investigation of $\overline{\mathcal{M}}_{g, n}$ becomes more natural when the whole collection of $\overline{\mathcal{M}}_{g, n}$ 's, and specific morphisms among them, is taken into account. By an elementary double induction argument, it is thus possible to prove various results

Received by the editors January 13, 2004 and, in revised form, September 8, 2004.

2000 Mathematics Subject Classification. Primary 14H10, 14E08.

(C)2006 American Mathematical Society 
on the rational cohomology of these spaces; see [17, 18] for related results which had been previously known via other methods.

Here we apply a similar induction argument to $\overline{\mathcal{M}}_{g, n}$ and $\bar{S}_{g, n}$. As a result, in Section 2 we derive some vanishings of Hodge numbers of type $(p, 0)$ for $\overline{\mathcal{M}}_{g, n}$. Next, in Section 3 we describe the rational Picard group of $\bar{S}_{g, n}$ and, as a corollary of [20, we determine generators and relations in the case $g \geq 9, n=0$. In Section 4 . we compute the Kodaira dimension of spin moduli spaces in several cases and leave the other ones as open questions, which we hope to address in the future with different methods. To carry through some of these calculations, we also compute the Kodaira dimension of $\overline{\mathcal{M}}_{1, n}$.

\section{2. $(p, 0)$ Hodge numbers of MOduli spaCes of Stable CURVES}

In [3], Enrico Arbarello and Maurizio Cornalba proved the vanishing of some odd cohomology groups of $\overline{\mathcal{M}}_{g, n}$. They applied an inductive method that reduces the problem to checking such vanishings for finitely many values of $g$ and $n$ in each odd degree $k$. Unfortunately, if $k \geq 11$ the inductive machinery does not work. Indeed, it is well known (see, for instance, 27, proof of Corollary 4.7, or [15, Proposition 2) that $h^{11,0}\left(\overline{\mathcal{M}}_{1,11}\right) \neq 0$, where $h^{p, q}\left(\overline{\mathcal{M}}_{g, n}\right)=\operatorname{dim} H^{p, q}\left(\overline{\mathcal{M}}_{g, n}\right)$ denote the Hodge numbers of $\overline{\mathcal{M}}_{g, n}$. On the other hand, from the results of $[3]$ it follows that $h^{p, 0}\left(\overline{\mathcal{M}}_{g, n}\right)=0$ for $p=1,3,5$. Here we complete the picture by showing that there are no non-zero $p$ holomorphic forms on $\overline{\mathcal{M}}_{g, n}$ for $0<p<11$. Precisely, the following holds.

Theorem 1. Let $g$ and $n$ be non-negative integers, $n>2-2 g$. If $0<p<11$, then $h^{p, 0}\left(\overline{\mathcal{M}}_{g, n}\right)=0$.

Proof. Let us consider the long exact sequence of cohomology with compact supports:

$$
\ldots \rightarrow H_{c}^{k}\left(\mathcal{M}_{g, n}\right) \rightarrow H^{k}\left(\overline{\mathcal{M}}_{g, n}\right) \rightarrow H^{k}\left(\partial \overline{\mathcal{M}}_{g, n}\right) \rightarrow \ldots
$$

From [18] we get the following vanishings of $H_{c}^{k}\left(\mathcal{M}_{g, n}\right)$ :

$$
k \leq d(g, n)= \begin{cases}n-4 & \text { if } g=0 \\ 2 g-2 & \text { if } n=0 \\ 2 g-3+n & \text { if } g>0, n>0\end{cases}
$$

Since the morphism

$$
H^{k}\left(\overline{\mathcal{M}}_{g, n}\right) \rightarrow H^{k}\left(\partial \overline{\mathcal{M}}_{g, n}\right)
$$

is compatible with the Hodge structures (see [3], p. 102), there is an injection

$$
H^{p, 0}\left(\overline{\mathcal{M}}_{g, n}\right) \hookrightarrow H^{p, 0}\left(\partial \overline{\mathcal{M}}_{g, n}\right)
$$

for $p \leq d(g, n)$.

As is well known, each irreducible component of $\partial \overline{\mathcal{M}}_{g, n}$ is the image of a map from $\overline{\mathcal{M}}_{g-1, n+2}$ or $\overline{\mathcal{M}}_{h, a+1} \times \overline{\mathcal{M}}_{g-h, b+1}$, where $0 \leq h \leq g, a+b=n$, and both $2 h-1+a$ and $2(g-h)-1+b$ are positive. By an analogue of Lemma (2.6) in [3], the map

$$
H^{p, 0}\left(\overline{\mathcal{M}}_{g, n}\right) \rightarrow H^{p, 0}\left(\overline{\mathcal{M}}_{g-1, n+2}\right) \oplus \bigoplus_{h, a} H^{p, 0}\left(\overline{\mathcal{M}}_{h, a+1} \times \overline{\mathcal{M}}_{g-h, b+1}\right)
$$


is injective whenever the map (1) is, and by the Künneth formula, the right-hand side of (2) involves only moduli spaces $\overline{\mathcal{M}}_{g^{\prime}, n^{\prime}}$ such that either $g^{\prime}<g$ or $n^{\prime}<n$. Hence the case $p \leq d(g, n)$ follows from a double induction argument on $g$ and $n$.

In order to conclude, it suffices to check that $h^{p, 0}\left(\overline{\mathcal{M}}_{g, n}\right)=0$ for every $p>$ $d(g, n)$. This is in fact the base of the previous induction. When $g=0$, the space $\overline{\mathcal{M}}_{0, n}$ is rational [25], so there is nothing to check. If $g=1$, we are dealing with $\overline{\mathcal{M}}_{1, n}$ for $n \leq 10$. As shown in [5], all these spaces are rational, so the base of the induction holds true. Finally, when $g \geq 2$ and $p>d(g, n), \overline{\mathcal{M}}_{g, n}$ is a unirational variety; see [26, Theorem 7.1. Hence, from a dominant rational map

$$
\mathbb{P}^{3 g-3+n} \rightarrow \overline{\mathcal{M}}_{g, n}
$$

we get an injective morphism

$$
H^{p, 0}\left(\overline{\mathcal{M}}_{g, n}\right) \hookrightarrow H^{p, 0}\left(\mathbb{P}^{3 g-3+n}\right)
$$

(as in [16], p. 494), and we have $h^{p, 0}\left(\mathbb{P}^{3 g-3+n}\right)=0$ for every $p>0$ (see for instance [16, Corollary on p. 118). Thus the claim is completely proved.

\section{The rational Picard group of SPin moduli spaces}

The moduli space $S_{g}$ of smooth spin curves is a classical object, which parametrizes pairs given by (smooth genus $g$ complex curve $C$, and theta-characteristic on $C$ ). Since it is a non-trivial covering of $\mathcal{M}_{g}$, a priori its geometry is much more complicated. However, John Harer in [19] and [20] succeeded in applying to $S_{g}$ his approach to the cohomology of moduli spaces via geometric topology. Furthermore, in [8] and [9], Maurizio Cornalba constructed a geometrically meaningful compactification $\bar{S}_{g}$ of $S_{g}$. Here we determine $\operatorname{Pic}\left(\bar{S}_{g, n}\right) \otimes \mathbb{Q}$ and give explicit generators and relations. For the reader's convenience we recall some basic definitions.

Let $X$ be a Deligne-Mumford semistable curve and let $E$ be a complete, irreducible subcurve of $X$. E is said to be exceptional when it is smooth, rational, and meets the other components in exactly two points. Moreover, $X$ is said to be quasi-stable when any two distinct exceptional components of $C$ are disjoint. In the sequel, $\tilde{X}$ will denote the subcurve $\overline{X \backslash \bigcup E_{i}}$ obtained from $X$ by removing all exceptional components.

A spin curve of genus $g$ (see $[\underline{8}, \S 2$ ) is the datum of a quasi-stable genus $g$ curve $X$ with an invertible sheaf $\zeta_{X}$ of degree $g-1$ on $X$ and a homomorphism of invertible sheaves

$$
\alpha_{X}: \zeta_{X}^{\otimes 2} \longrightarrow \omega_{X}
$$

such that

(i) $\zeta_{X}$ has degree 1 on every exceptional component of $X$;

(ii) $\alpha_{X}$ is not zero at a general point of every non-exceptional component of $X$. Therefore, $\alpha_{X}$ vanishes identically on all exceptional components of $X$ and induces an isomorphism

$$
\tilde{\alpha}_{X}:\left.\zeta_{X}^{\otimes 2}\right|_{\tilde{X}} \longrightarrow \omega_{\tilde{X}}
$$

In particular, when $X$ is smooth, $\zeta_{X}$ is just a theta-characteristic on $X$.

Two spin curves $\left(X, \zeta_{X}, \alpha_{X}\right)$ and $\left(X^{\prime}, \zeta_{X^{\prime}}, \alpha_{X^{\prime}}\right)$ are isomorphic if there are isomorphisms $\sigma: X \rightarrow X^{\prime}$ and $\tau: \sigma^{*}\left(\zeta_{X}^{\prime}\right) \rightarrow \zeta_{X}$ such that $\tau$ is compatible with the natural isomorphism between $\sigma^{*}\left(\omega_{X^{\prime}}\right)$ and $\omega_{X}$. 
A family of spin curves is a flat family of quasi-stable curves $f: \mathcal{X} \rightarrow S$ with an invertible sheaf $\zeta_{f}$ on $\mathcal{X}$ and a homomorphism

$$
\alpha_{f}: \zeta_{f}^{\otimes 2} \longrightarrow \omega_{f}
$$

such that the restriction of these data to any fiber of $f$ gives rise to a spin curve.

Two families of spin curves $f: \mathcal{X} \rightarrow S$ and $f^{\prime}: \mathcal{X}^{\prime} \rightarrow S$ are isomorphic if there are isomorphisms $\sigma: \mathcal{X} \longrightarrow \mathcal{X}^{\prime}$ and $\tau: \sigma^{*}\left(\zeta_{f^{\prime}}\right) \longrightarrow \zeta_{f}$ such that $f=f^{\prime} \circ \sigma$ and $\tau$ is compatible with the natural isomorphism between $\sigma^{*}\left(\omega_{f^{\prime}}\right)$ and $\omega_{f}$.

Let $\bar{S}_{g}$ be the set of isomorphism classes of spin curves of genus $g$ and let $S_{g}$ be the subset consisting of classes of smooth curves. One can define a natural structure of analytic variety on $\bar{S}_{g}$ (see [8], $\S 5$ ) in such a way that for any spin curve $X$ there is a neighbourhood of $[X]$ in $\bar{S}_{g}$ and an isomorphism

$$
U \cong B_{X} / \operatorname{Aut}(X)
$$

where $B_{X}$ is a $3 g-3$-dimensional polydisk and $\operatorname{Aut}(X)$, the group of automorphisms of the spin curve $X$, is a finite group (see [8], Lemma (2.2)). These spaces can be slightly generalized as follows:

$$
\begin{aligned}
\bar{S}_{g, n}:= & \left\{\left[\left(C, p_{1}, \ldots, p_{n} ; \zeta ; \alpha\right)\right]:\left(C, p_{1}, \ldots, p_{n}\right) \text { is a genus } g\right. \\
& \text { quasi-stable projective curve with } n \text { marked points; } \\
& \zeta \text { is a line bundle of degree } g-1 \text { on } C \text { having } \\
& \text { degree } 1 \text { on every exceptional component of } C, \text { and } \\
& \alpha: \zeta^{\otimes 2} \rightarrow \omega_{C} \text { is a homomorphism which is } \\
& \text { not zero at a general point of every non-exceptional } \\
& \text { component of } C\} .
\end{aligned}
$$

Analogously to $\bar{S}_{g}$, these spaces are normal projective varieties of complex dimension $3 g-3+n$ with finite quotient singularities. We point out the following fact.

Lemma 1. Let $\operatorname{Pic}\left(\bar{S}_{g, n}\right):=H^{1}\left(\bar{S}_{g, n}, \mathcal{O}^{*}\right)$. There is a natural isomorphism

$$
\operatorname{Pic}\left(\bar{S}_{g, n}\right) \otimes \mathbb{Q} \stackrel{\cong}{\cong} A_{3 g-4+n}\left(\bar{S}_{g, n}\right) \otimes \mathbb{Q} .
$$

Proof. Since $\bar{S}_{g, n}$ is normal (see [8], Proposition (5.2)), there is an injection:

$$
\operatorname{Pic}\left(\bar{S}_{g, n}\right) \hookrightarrow A_{3 g-4+n}\left(\bar{S}_{g, n}\right) .
$$

Moreover, from the construction of $\bar{S}_{g, n}$ it follows that the singularities of $\bar{S}_{g, n}$ are of finite quotient type, so every Weil divisor is $\mathbb{Q}$-Cartier and there is a surjective morphism:

$$
\operatorname{Pic}\left(\bar{S}_{g, n}\right) \otimes \mathbb{Q} \rightarrow A_{3 g-4+n}\left(\bar{S}_{g, n}\right) \otimes \mathbb{Q} .
$$

Hence the claim follows.

When $n=0$, it is also possible to give a more precise description of of $\operatorname{Pic}\left(\bar{S}_{g}\right) \otimes \mathbb{Q}$. To this end, recall that $\bar{S}_{g}$ is the disjoint union of two irreducible subvarieties $\bar{S}_{g}^{+}$ and $\bar{S}_{g}^{-}$which consist of the even and the odd spin curves of genus $g$ (see 8 , Lemma (6.3)), respectively. The following crucial result was obtained by John Harer via geometric topology (see [20], Corollary 1.3). 
Theorem 2. Let $\mathcal{M}_{g}[\varepsilon]$ denote either $\bar{S}_{g}^{+} \cap S_{g}$ or $\bar{S}_{g}^{-} \cap S_{g}$. Then $\operatorname{Pic}\left(\mathcal{M}_{g}[\varepsilon]\right) \otimes \mathbb{Q}:=$ $H^{1}\left(\mathcal{M}_{g}[\varepsilon], \mathcal{O}^{*}\right) \otimes \mathbb{Q}$ has rank 1 for $g \geq 9$.

For any family $f: \mathcal{X} \rightarrow S$ of spin curves, $M_{f}:=\operatorname{det} R f_{*} \zeta_{f}$ is a line bundle on $S$. Let $M$ denote the corresponding line bundle on $\bar{S}_{g}$ associated to the universal family on $\bar{S}_{g}$ (as usual, note that for $g \geq 4$ the locus of spin curves with automorphisms has complex codimension $\geq 2)$. Let $\mu^{+}\left(\right.$resp. $\left.\mu^{-}\right)$be the class of $M \operatorname{in} \operatorname{Pic}\left(\bar{S}_{g}^{+}\right)$ (resp. Pic $\left(\bar{S}_{g}^{-}\right)$. The boundary $\partial \bar{S}_{g}=\bar{S}_{g} \backslash S_{g}$ is the union of irreducible components $A_{i}^{+}, B_{i}^{+}$(contained in $\bar{S}_{g}^{+}$) and $A_{i}^{-}, B_{i}^{-}$(contained in $\bar{S}_{g}^{-}$), which are completely described in [8], $\S 7$. Let $\alpha_{i}^{+}, \beta_{i}^{+}, \alpha_{i}^{-}, \beta_{i}^{-}$denote the corresponding classes in $A_{3 g-4}\left(\bar{S}_{g}\right)$. The following result is contained in [8], Proposition (7.2).

Proposition 1. If $g>2$ is odd, the classes $\mu^{+}, \mu^{-}, \alpha_{i}^{+}, \alpha_{i}^{-}, \beta_{i}^{+}, \beta_{i}^{-}, i=$ $0, \ldots,(g-1) / 2$, are independent. If $g>2$ is even, the same is true of the classes $\mu^{+}, \mu^{-}, \alpha_{i}^{+}, \alpha_{i}^{-}, \beta_{i}^{+}, i=0, \ldots, g / 2$, and $\beta_{i}^{-}, i=0, \ldots, g / 2-1$.

Now we are ready to state and prove a description by generators and relations of the rational Picard group of $\bar{S}_{g}$ :

Corollary 1. Assume $g \geq 9$. If $g$ is odd, then $\operatorname{Pic}\left(\bar{S}_{g}^{+}\right)$(resp. Pic $\left(\bar{S}_{g}^{-}\right)$) is freely generated over $\mathbb{Q}$ by the classes $\mu^{+}, \alpha_{i}^{+}, \beta_{i}^{+}, i=0, \ldots,(g-1) / 2$ (resp. by the classes $\left.\mu^{-}, \alpha_{i}^{-}, \beta_{i}^{-}, i=0, \ldots,(g-1) / 2\right)$. If $g$ is even, then $\operatorname{Pic}\left(\bar{S}_{g}^{+}\right)\left(\operatorname{resp} . \operatorname{Pic}\left(\bar{S}_{g}^{-}\right)\right)$is freely generated over $\mathbb{Q}$ by the classes $\mu^{+}, \alpha_{i}^{+}, \beta_{i}^{+}, i=0, \ldots, g / 2$ (resp. by the classes $\mu^{-}, \alpha_{i}^{-}, i=0, \ldots, g / 2$, and $\left.\beta_{i}^{-}, i=0, \ldots, g / 2-1\right)$.

Proof. By Lemma 1 we may use the exact sequence

$$
A_{3 g-4}\left(\bar{S}_{g} \backslash S_{g}\right) \rightarrow A_{3 g-4}\left(\bar{S}_{g}\right) \rightarrow A_{3 g-4}\left(S_{g}\right) \rightarrow 0
$$

to conclude that $\operatorname{Pic}\left(\bar{S}_{g}\right) \otimes \mathbb{Q}$ is generated by the generators of $A_{3 g-4}\left(S_{g}\right)$ together with the set of boundary classes of $\bar{S}_{g}$. From Theorem 2 it follows that $\operatorname{Pic}\left(S_{g}\right) \otimes \mathbb{Q}$ is generated by the classes $\mu^{+}$and $\mu^{-}$, therefore $\operatorname{Pic}\left(\bar{S}_{g}\right) \otimes \mathbb{Q}$ is generated by the classes $\mu^{+}, \mu^{-}, \alpha_{i}^{+}, \alpha_{i}^{-}, \beta_{i}^{+}, \beta_{i}^{-}$. By Proposition 1, all these classes are independent, so Corollary 1 is proved.

\section{Calculation of Kodaira dimensions}

In this section, we calculate the Kodaira dimension of some spin moduli spaces. We recall that the Kodaira dimension is an important birational invariant in the classification of projective varieties. As a first general result, we prove the following.

Proposition 2. Fix $g$ and $n$ non-negative integers, $n>2-2 g$. Any irreducible component of $\bar{S}_{g, n}$ is of general type whenever $\overline{\mathcal{M}}_{g, n}$ is.

Proof. Since any component of $\bar{S}_{g, n}$ is a non-trivial ramified covering of $\overline{\mathcal{M}}_{g, n}$, then the claim follows from [28], Theorem 6.10.

In particular, $\bar{S}_{g}$ is of general type for $g \geq 24$. When $n \geq 1$, Logan determines in [26] integers $\widetilde{n}(g), g \geq 2$, such that $\overline{\mathcal{M}}_{g, n}$ is of general type when $n \geq \widetilde{n}(g)$. As pointed out in Section 3, when $g=0$, spin moduli spaces are rational since they are isomorphic to $\overline{\mathcal{M}}_{0, n}$. To tackle the genus one case, we first need to compute the Kodaira dimension of $\overline{\mathcal{M}}_{1, n}$, which does not seem to be thoroughly dealt with in the literature. It turns out that $\kappa\left(\overline{\mathcal{M}}_{1, n}\right)$ varies with the number of marked points. 
As proved in [5], the moduli space of $n$-pointed genus 1 curves is rational for $n \leq 10$, hence $\kappa\left(\overline{\mathcal{M}}_{1, n}\right)=-\infty$. To compute the Kodaira dimension for $n \geq 11$, we first need to express the canonical divisor $K_{\overline{\mathcal{M}}_{1, n}}$ in terms of generators of the rational Picard group of $\overline{\mathcal{M}}_{1, n}$. We briefly recall such generators and some of their relations: for more details the reader is referred, for instance, to [2].

As usual, we denote by $\lambda$ the first Chern class of the Hodge bundle whose fiber over the element $\left[C ; p_{1}, \ldots, p_{n}\right] \in \overline{\mathcal{M}}_{1, n}$ is $H^{0}\left(C, \omega_{C}\right)$, where $\omega_{C}$ is the dualizing sheaf of $C$. Next, we denote by $\psi_{i}, 1 \leq i \leq n$, the first Chern class of the line bundle whose fiber over $\left[C ; p_{1}, \ldots, p_{n}\right]$ is the cotangent space of $C$ at the (smooth) point $p_{i}$. Finally, we denote by $\delta_{i r r}$ and $\delta_{0, S}$ the classes corresponding to boundary divisors. Here $\delta_{i r r}$ is the (rational) Poincaré dual of the locus of curves with one non-disconnecting node and $n$ marked points. The class $\delta_{0, S},|S| \geq 2$, corresponds the locus of curves with a disconnecting node whose removal creates two connected components: one of genus 0 with the marked points labelled by the elements of $S$ and the other one of genus 1 with the marked points labelled by the elements of $S^{c}$. Last, note that by $\kappa_{m}$ and $\widetilde{\kappa}_{m}, m \geq 1$, we mean the Mumford classes as described in 3 .

We finally recall that $\operatorname{Pic}\left(\overline{\mathcal{M}}_{g, n}\right) \otimes \mathbb{Q}$ is isomorphic to the Picard group of the moduli stack of $n$-pointed genus $g$ stable curves. We shall first compute the canonical class of this stack so as to deduce $K_{\overline{\mathcal{M}}_{1, n}}$.

Proposition 3. For any non-negative integer $n \geq 3$,

$$
\begin{aligned}
& K_{\overline{\mathcal{M}}_{1, n}}=(n-11) \lambda+(n-3) \delta_{0,\{1, \ldots, n\}} \\
&+ \sum_{\substack{|S| \geq 2 \\
S \subset\{1, \ldots, n\}}}(|S|-2) \delta_{0, S} .\{1, \ldots, n\} \\
&
\end{aligned}
$$

Proof. Suppose $\rho: \mathcal{C} \rightarrow B$ is a family of $n$-pointed genus 1 curves over a smooth base $B$ and with general smooth fiber. Let $\sigma_{1}, \ldots, \sigma_{n}$ denote the $n$ canonical sections and define $D$ to be the sum of the divisors corresponding to them. As in 21, we apply Grothendieck-Riemann-Roch Theorem to the bundle $\Omega_{\mathcal{C} / B}^{1}(D) \otimes \omega_{\mathcal{C} / B}$, where $\Omega_{\mathcal{C} / B}^{1}$ is the sheaf of relative Kähler differentials and $\omega_{\mathcal{C} / B}$ is the relative dualizing sheaf of $\rho$. Therefore,

$$
\begin{aligned}
K & =c_{1}\left(\rho_{*}\left(\Omega_{\mathcal{C} / B}^{1}(D) \otimes \omega_{\mathcal{C} / B}\right)\right) \\
& =\rho_{*}\left(T d_{2}^{\vee}\left(\Omega_{\mathcal{C} / B}^{1}\right)+\operatorname{ch}_{2}\left(\Omega_{\mathcal{C} / B}^{1}(D) \otimes \omega_{\mathcal{C} / B}\right)\right) \\
& +\rho_{*}\left(T d_{1}^{\vee}\left(\Omega_{\mathcal{C} / B}^{1}\right) \operatorname{ch}_{1}\left(\Omega_{\mathcal{C} / B}^{1}(D) \otimes \omega_{\mathcal{C} / B}\right)\right),
\end{aligned}
$$

where $T d_{j}^{\vee}$ and $c h_{j}$ denote the degree $j$ term of the Todd class $T d^{\vee}$ and of the Chern character $c h$, respectively.

If $\eta$ is the class of the locus of nodes of fibers of $\mathcal{C}$ over $B$, we have

$$
\begin{gathered}
T d_{1}^{\vee}\left(\Omega_{\mathcal{C} / B}^{1}\right)=-\frac{1}{2} c_{1}\left(\omega_{\mathcal{C} / B}\right), \\
T d_{2}^{\vee}\left(\Omega_{\mathcal{C} / B}^{1}\right)=\frac{1}{12}\left(c_{1}^{2}\left(\omega_{\mathcal{C} / B}\right)+\eta\right) .
\end{gathered}
$$


Analogously,

$$
\begin{aligned}
\operatorname{ch}_{1}\left(\Omega_{\mathcal{C} / B}^{1}(D) \otimes \omega_{\mathcal{C} / B}\right) & =c_{1}\left(\omega_{\mathcal{C} / B}(D)\right)+c_{1}\left(\omega_{\mathcal{C} / B}\right) \\
\left.c h_{2}\left(\Omega_{\mathcal{C} / B}^{1}(D) \otimes \omega_{\mathcal{C} / B}\right)\right) & =\frac{1}{2} c_{1}^{2}\left(\omega_{\mathcal{C} / B}\right)-\eta \\
& +c_{1}\left(\omega_{\mathcal{C} / B}(D)\right) c_{1}\left(\omega_{\mathcal{C} / B}\right)+\frac{1}{2} c_{1}^{2}\left(\omega_{\mathcal{C} / B}(D)\right) .
\end{aligned}
$$

By the definition of $\kappa_{1}$ and $\widetilde{\kappa}_{1}$, we have

$$
\begin{aligned}
K & =\frac{1}{12} \widetilde{\kappa}_{1}+\frac{1}{2} \rho_{*}\left(c_{1}\left(\omega_{\mathcal{C} / B}(D)\right) c_{1}\left(\omega_{\mathcal{C} / B}\right)\right) \\
& -\frac{11}{12} \rho_{*}(\eta)+\frac{1}{2} \kappa_{1} .
\end{aligned}
$$

On the other hand, by [4,

$$
\kappa_{1}=\widetilde{\kappa}_{1}+\sum_{i=1}^{n} \psi_{i}
$$

and

$$
\frac{1}{2} \rho_{*}\left(c_{1}\left(\omega_{\mathcal{C} / B}(D)\right) c_{1}\left(\omega_{\mathcal{C} / B}\right)\right)=\frac{1}{2} \widetilde{\kappa}_{1}+\sum_{i=1}^{n} \psi_{i} .
$$

Therefore, by Example 2.1 in [6], we have

$$
K=13 \lambda+\sum_{i=1}^{n} \psi_{i}-2 \delta
$$

where

$$
\delta:=\delta_{i r r}+\sum_{\substack{|S| \geq 2 \\ S \subset\{1, \ldots, n\}}} \delta_{0, S}
$$

Moreover, in genus 1 (see [3]), this can be rewritten as

$$
K=(n-11) \lambda+\sum_{\substack{|S| \geq 2 \\ S \subset\{1, \ldots, n\}}}(|S|-2) \delta_{0, S} .
$$

Since the map from the moduli stack of $n$-pointed, $n \geq 3$, genus 1 curves to the (coarse) moduli space is ramified along the divisor $\delta_{0,\{1, \ldots, n\}}$, the claim follows.

Remark 1. When $g=1$ and $n=1$, the (coarse) moduli space is isomorphic to $\mathbb{P}^{1}$, so the canonical class is known. When $g=1$, and $n=2$, then (77) simplifies to $-9 \lambda$. Analogously to the case $n \geq 3$, the canonical class is $-9 \lambda-\delta_{0,\{1,2\}}$.

Remark 2. In [26], a formula for the canonical divisor of $\overline{\mathcal{M}}_{g, n}$ is given. The proof relies on the corresponding formula for $K_{\overline{\mathcal{M}}_{g}}$, the canonical divisor of $\overline{\mathcal{M}}_{g}$, obtained in 22] only for $g \geq 2$ via Grothendieck-Riemann-Roch Theorem. To the authors' knowledge, an analogous formula in genus 1 is not explicitely stated in the literature.

We can now complete the computation of $\kappa\left(\overline{\mathcal{M}}_{1, n}\right)$ for each $n \geq 1$. In fact, the following holds. 
Theorem 3. We have

$$
\kappa\left(\overline{\mathcal{M}}_{1, n}\right)= \begin{cases}0, & n=11 \\ 1, & n \geq 12 .\end{cases}
$$

Proof. By Proposition [3, $K_{\overline{\mathcal{M}}_{1,11}}$ is an effective divisor, hence $\kappa\left(\overline{\mathcal{M}}_{1,11}\right) \geq 0$. On the other hand, $\mathcal{M}_{1,11}$ is birational to a hypersurface $X$ of $\left(\mathbb{P}^{2}\right)^{6}$ of multidegree $(3, \ldots, 3)$ (see Remark 1.2.4 in [5]). By adjunction, we obtain that $K_{X}$ is trivial. In order to compute the Kodaira dimension of $X$, let $f: Y \rightarrow X$ be the normalization map. We have $K_{Y}=f^{*} K_{X}-\Delta$, where the conductor $\Delta$ is an effective divisor. It follows that $\kappa\left(\overline{\mathcal{M}}_{1,11}\right)=\kappa(X)=\kappa(Y) \leq 0$, so the case $n=11$ is over.

Next, again by Proposition $3, K_{\overline{\mathcal{M}}_{1, n}}, n \geq 12$, is the sum of two effective divisors, i.e., $L:=(n-11) \lambda$ and

$$
E:=(n-3) \delta_{0,\{1, \ldots, n\}}+\sum_{\substack{|S| \geq 2, S \neq\{1, \ldots, n\} \\ S \subset\{1, \ldots, n\}}}(|S|-2) \delta_{0, S} .
$$

Therefore, the Kodaira dimension of $\overline{\mathcal{M}}_{1, n}$ is greater than or equal to the Iitaka dimension of the divisor $L$. On the other hand, let

$$
\pi: \overline{\mathcal{M}}_{1, n} \rightarrow \overline{\mathcal{M}}_{1,1}
$$

be the morphism which forgets the last $n-1$ points and passes to the stable model. If $\lambda_{1}$ denotes the first Chern class of the Hodge bundle on $\overline{\mathcal{M}}_{1,1}$, then we have $\lambda=\pi^{*}\left(\lambda_{1}\right)$ (see for instance [2], (6)). Moreover, since $\lambda_{1}$ is ample on $\overline{\mathcal{M}}_{1,1}$, we obtain

$$
\kappa\left(\overline{\mathcal{M}}_{1, n}, L\right) \geq \kappa\left(\overline{\mathcal{M}}_{1,1},(n-11) \lambda_{1}\right)=1 .
$$

This proves that $\kappa\left(\overline{\mathcal{M}}_{1, n}\right) \geq 1$. However, the fiber of $[C ; p] \in \overline{\mathcal{M}}_{1, n}$ under $\pi$ can be viewed as the quotient by a finite group of an open Zariski subset of the product

$$
\underbrace{C \times \ldots \times C}_{(n-1) \text { times }},
$$

hence $\kappa\left(\pi^{-1}([C ; p])\right) \leq 0$. By Theorem 6.12 in $[28$, it follows that

$$
\kappa\left(\overline{\mathcal{M}}_{1, n}\right) \leq \kappa\left(\pi^{-1}([C ; p])\right)+\operatorname{dim}\left(\overline{\mathcal{M}}_{1,1}\right) \leq 1 .
$$

Thus the claim is completely proved.

Corollary 2. For any $n \geq 1, \overline{\mathcal{M}}_{1, n}$ is never of general type.

Next, we turn to moduli spaces of pointed spin curves of genus 1. Recall from Section 3 that $\bar{S}_{1, n}$ is the compactification $\grave{a}$ la Deligne-Mumford of the moduli space of $n$-pointed smooth elliptic curves with a theta-characteristic. Note that $\bar{S}_{1, n}$ is the disjoint union of $\bar{S}_{1, n}^{+}$and $\bar{S}_{1, n}^{-}$, which correspond to even and odd theta-characteristics, respectively. However, since over an elliptic curve there is only one odd theta-characteristic (namely, the structural sheaf), there is a natural isomorphism $\bar{S}_{1, n}^{-} \cong \overline{\mathcal{M}}_{1, n}$. From now on, we thus focus our attention on $\bar{S}_{1, n}^{+}$.

In order to prove that $\kappa\left(\bar{S}_{1, n}^{+}\right)=-\infty$ for $n \leq 10$, we are going to show that $\bar{S}_{1, n}^{+}$ is uniruled whenever $\overline{\mathcal{M}}_{1, n}$ is. Indeed, the following holds.

Lemma 2. Let $p: \bar{S}_{1, n}^{+} \rightarrow \overline{\mathcal{M}}_{1, n}$ denote the natural projection. If $C$ is a rational curve in $\overline{\mathcal{M}}_{1, n}$, then there exists a rational curve $D$ in $\bar{S}_{1, n}^{+}$such that $p(D)=C$. 
Proof. The proof is by induction on $n$.

If $n=1$, then we have $\overline{\mathcal{M}}_{1, n} \cong \mathbb{P}^{1}$ and $\bar{S}_{1, n}^{+} \cong \mathbb{P}^{1}$, so in this case the stated property is obvious.

Assume now that $n>1$. It is easy to check that

$$
\bar{S}_{1, n}^{+}=\overline{\mathcal{M}}_{1, n} \times \overline{\mathcal{M}}_{1, n-1} \bar{S}_{1, n-1}^{+} .
$$

Therefore, we have the following commutative diagram:

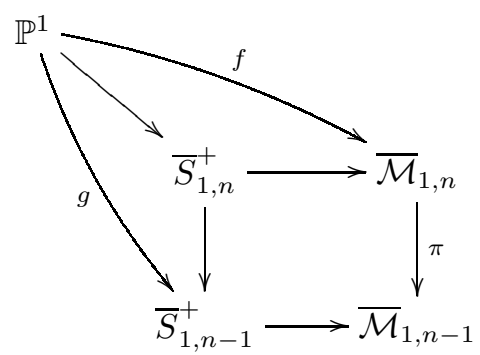

Diagram 1. Uniruledness of spin moduli spaces for $g=1, n \leq 10$.

In Diagram $1, f$ exists by hypothesis and $g$ exists by the inductive assumption (just note that $\pi \circ f\left(\mathbb{P}^{1}\right)$ is not a point since the fibers of $\pi$ do not contain rational curves). Hence the claim follows from the universal property of the fibered product.

Finally, we consider the case $n \geq 12$.

Proposition 4. Let $n \geq 12$ be a non-negative integer. Then the Kodaira dimension of $\bar{S}_{1, n}^{+}$is 1 .

Proof. Since the natural projection

$$
p: \bar{S}_{1, n}^{+} \longrightarrow \overline{\mathcal{M}}_{1, n}
$$

is a surjective map between normal varieties of the same dimension, it follows that

$$
\kappa\left(\bar{S}_{1, n}^{+}\right) \geq \kappa\left(\overline{\mathcal{M}}_{1, n}\right)=1
$$

(see 28, Theorem 6.10).

On the other hand, the fiber of the forgetful map

$$
\bar{S}_{1, n}^{+} \longrightarrow \bar{S}_{1,1}^{+}
$$

is precisely the same as that of the morphism (8). Hence, exactly as in the proof of Theorem 3, we can deduce that

$$
\kappa\left(\bar{S}_{1, n}^{+}\right) \leq 0+\operatorname{dim}\left(\bar{S}_{1,1}^{+}\right) \leq 1 .
$$

Thus the proof follows.

By the same arguments as in Proposition 4, we get the estimate

$$
0 \leq \kappa\left(\bar{S}_{1,11}^{+}\right) \leq 1 \text {. }
$$

Another reason why $\kappa\left(\bar{S}_{1,11}^{+}\right) \geq 0$ is given by the following result.

Proposition 5. There exists a non-zero holomorphic form of degree 11 on $\bar{S}_{1, n}^{+}$. 
Proof. Since the natural projection

$$
p: \bar{S}_{1, n}^{+} \longrightarrow \overline{\mathcal{M}}_{1, n}
$$

is a surjective map between normal varieties of the same dimension, the induced map

is injective.

$$
p^{*}: H^{11,0}\left(\overline{\mathcal{M}}_{1, n}, \mathbb{Q}\right) \rightarrow H^{11,0}\left(\bar{S}_{1, n}^{+}, \mathbb{Q}\right)
$$

We end this section with a couple of natural questions.

Question 1. Is the Kodaira dimension of $\bar{S}_{1,11}^{+}$zero?

Question 2. Is any irreducible component of $\bar{S}_{g, n}$ unirational whenever the corresponding moduli space $\overline{\mathcal{M}}_{g, n}$ is?

\section{ACKNOWLEDGEMENTS}

The authors would like to thank M. Andreatta, E. Ballico and S. Keel for useful remarks and suggestions.

\section{REFERENCES}

[1] Abramovich, D., Jarvis, T. J.: Moduli of twisted spin curves. Proc. Amer. Math. Soc. 131, 685-699 (2003). MR1937405 (2003k:14027)

[2] Arbarello, E., Cornalba, M.D.T.: The Picard groups of the moduli spaces of curves. Topology 26, 153-171 (1987). MR0895568 (88e:14032)

[3] Arbarello, E., Cornalba, M.D.T.: Calculating cohomology groups of moduli spaces of curves via algebraic geometry. Inst. Hautes Études Sci. Publ. Math. 88, 97-127 (1998). MR1733327 (2001h:14030)

[4] Arbarello, E., Cornalba, M.D.T.: Combinatorial and algebro-geometric cohomology classes on the moduli spaces of curves. J. Alg. Geom. 5, 705-749 (1996). MR1486986 (99c:14033)

[5] Belorousski, P.: Chow rings of moduli spaces of pointed elliptic curves. Ph.D. thesis, Chicago, 1998.

[6] Bini, G.: Generalized Hodge classes on moduli spaces of curves. Beiträge zur Algebra und Geometrie 44 (2003), 559-565. MR2017857 (2004k:14042)

[7] Caporaso, L., Casagrande, C.: Combinatorial properties of stable spin curves. Special issue in honor of Steven L. Kleiman. Comm. Algebra 31 (2003), no. 8, 3653-3672. MR2007379 (2004i:14026)

[8] Cornalba, M.D.T.: Moduli of curves and theta-characteristics. In: Lectures on Riemann surfaces (Trieste, 1987), 560-589, Teaneck, N.J.: World Sci. Publishing, 1989. MR.1082361 (91m:14037)

[9] Cornalba, M.D.T.: A remark on the Picard group of spin moduli space. Rend. Mat. Acc. Lincei (9) Mat. Appl. 2, 211-217 (1991). MR.1135424 (92k:14007)

[10] Cornalba, M.D.T.: Cohomology of moduli spaces of stable curves. In: Proceedings of the International Congress of Mathematicians, Vol. II (Berlin, 1998), 249-257, Doc. Math., 1998. MR.1648075 (99i:14031)

[11] Faber, C., Looijenga, E., Remarks on moduli of curves. In: Moduli of curves and abelian varieties, 23-45, Aspects Math., E33, Vieweg, Braunschweig, 1999. MR1722537(2001b:14044)

[12] Farkas, G.: The geometry of the moduli space of curves of genus 23. Math. Ann. 318, 43-65 (2000). MR 1785575 (2001f:14048)

[13] Getzler, E.: The semi-classical approximation for modular operads. Comm. Math. Phys. 194, 481-492 (1998). MR:1627677|(99d:14017)

[14] Getzler, E.: The Hodge polynomials of $\overline{\mathcal{M}}_{3,1}$. Preprint http://xxx.lanl.gov/abs/ math.AG/9910174 (1999).

[15] Graber, T., Pandharipande, R.: Constructions of nontautological classes on moduli spaces of curves. Michigan Math. J. 51, 93-109 (2003). MR1960923 (2004e:14043) 
[16] Griffiths, P., Harris, J.: Principles of Algebraic Geometry. New York: John Wiley and Sons, Inc., 1978. MR0507725 (80b:14001)

[17] Harer, J.: The second homology group of the mapping class group of an orientable surface. Inv. Math. 72, 221-239 (1982). MR0700769 (84g:57006)

[18] Harer, J.: The virtual cohomological dimension of the mapping class group of an orientable surface. Invent. Math. 84, 157-176 (1986). MR0830043 (87c:32030)

[19] Harer, J.: Stability of the homology of the moduli spaces of Riemann surfaces with spin structure. Math. Ann. 287, 323-334 (1990). MR1054572 (91e:57002)

[20] Harer, J.: The rational Picard group of the moduli space of Riemann surfaces with spin structure. In: Mapping class groups and moduli spaces of Riemann surfaces (Göttingen, 1991/Seattle, WA, 1991), 107-136, Contemp. Math. 150, 1993. MR.1234262 (94h:14008)

[21] Harris, J., Morrison, I.: Moduli of curves, Springer-Verlag, New York, 1998. MR1631825 (99g:14031)

[22] Harris, J., Mumford, D.: On the Kodaira dimension of the moduli space of curves. With an Appendix by William Fulton. Invent. Math. 67 (1982), 23-88. MR0664324 (83i:14018)

[23] Jarvis, T.J.: Geometry of the moduli of higher spin curves. Internat. J. Math. 11, 637-663 (2000). MR 1780734 (2001f:14050)

[24] Jarvis, T.J.: Picard group of the moduli of higher spin curves. New York J. Math. 7, 23-47 (2001). MR 1838471 (2002j:14032)

[25] Keel, S.: Intersection theory of moduli space of stable $n$ pointed curves of genus zero. Trans. Amer. Math. Soc. 330 (1992), 545-574. MR.1034665 (92f:14003)

[26] Logan, A.: The Kodaira dimension of moduli spaces of curves with marked points. Amer. J. Math. 125, 105-137 (2003). MR1953519 (2003j:14035)

[27] Pikaart, M.: An orbifold partition of $\bar{M}_{g}^{n}$. In: The moduli space of curves, (Texel Island, 1994), Progr. Math. 129, 467-482. Birkhäuser, Boston, 1995. MR1363067 (96m:14033)

[28] Ueno, U.: Classification theory of algebraic varieties and compact complex spaces, Lec. Notes in Math. 439, Springer-Verlag, New York, 1975. MR0506253 (58:22062)

Dipartimento di Matematica, Università degli Studi di Milano, Via C. Saldini 50, 20133 Milano, Italy

E-mail address: gilberto.bini@mat.unimi.it

Dipartimento di Matematica, Politecnico di Torino, Corso Duca degli Abruzzi 24, 10129 TORINO, ITALY

E-mail address: fontanar@science.unitn.it 